\title{
Effect of Fatigue on Electromyographic Activity Patterns of the Knee Joint Muscles in Anterior Cruciate Ligament Reconstructed and Deficient Patients during Landing Task
}

\author{
Komeil Dashti Rostami ${ }^{1}$, , Mohammad Hossein Alizadeh ${ }^{1}$, Hooman Minoonejad ${ }^{1}$, \\ Hamidreza Yazdi ${ }^{2}$ and Abbey Thomas ${ }^{3}$ \\ 1 Department of health and sports medicine, Faculty of physical education and sports sciences, \\ University of Tehran, 1417614418 Tehran, Iran; alizadehm@ut.ac.ir (M.H.A.); h.minoonejad@ut.ac.ir (H.M.) \\ 2 Neuromusculoskeletal research center, Iran University of Medical Sciences, 1449614535 Tehran, Iran; \\ yazdi.hr@iums.ac.ir \\ 3 Department of Kinesiology, University of North Carolina at Charlotte, Charlotte, NC 28223, USA; \\ afenwick@uncc.edu \\ * Correspondence: komeil.dashti@ut.ac.ir; Tel.: +98-9111-559-083
}

Received: 28 February 2018; Accepted: 3 April 2018; Published: 5 April 2018

\begin{abstract}
Lower extremity fatigue causes a number of neuromuscular alterations that may increase the risk of knee (re)injury. It has therefore been suggested that fatigue-resistance training be incorporated into injury prevention and rehabilitation programs. The purpose of this study was to determine the effects of fatigue on knee-muscle activity patterns after anterior cruciate ligament injury (ACL). Twelve healthy, 12 ACL reconstructed (ACLR) and 12 ACL deficient (ACLD) recreationally active male volunteers participated in this case-control study. Fatigue was induced via repetitive sets of double-leg squats and drop landings. Muscle activity was assessed via surface electromyography during a single-leg drop landing task pre- and post-fatigue. After fatigue, medial hamstring, lateral hamstring and vastus lateralis muscles turned on significantly earlier in ACLR compared to the control group. Significantly greater vastus medialis muscle activity was observed in ACLD compared to ACLR and control participants post-fatigue. Finally, fatigue decreased lateral gastrocnemius muscle activity in ACLD and control participants. It seems ACLR patients rely on earlier muscle pre-activation to establish knee joint stability upon landing after fatigue. This feed-forward strategy should be emphasized during post-operative rehabilitation in ACLR patients. Furthermore, according to our results, quadriceps and gastrocnemius muscles play an important role in establishing knee joint stability post impact in ACLD patients.
\end{abstract}

Keywords: knee; injury; neuromuscular control; rehabilitation

\section{Introduction}

Two major impacts of anterior cruciate ligament (ACL) rupture, whether treated conservatively or surgically, are failure to return to pre-injury activity levels [1-4] and future predisposition to osteoarthritis [5]. In fact, up to $48 \%$ of individuals do not return to their pre-injury sporting levels [1], and approximately half will go on to develop osteoarthritis [6]. Aberrant neuromuscular and poor biomechanical patterns are commonly seen up to two years after ACL injury/reconstruction and may help explain low successful return to pre-injury activity level, the high rate of second ACL injury and progression of osteoarthritis [6,7].

Some studies have shown that patients after ACL reconstruction (ACLR) and those who remain ACL deficient (ACLD) activate their lower extremity muscles earlier than healthy people [8-11]. 
The onset of muscle activity before landing is particularly of interest because it increases the stiffness of the joints [12]. This feed-forward mechanism is important as it allows the muscles time to generate force to provide correct lower extremity alignment during landing. Insufficient timing may place the knee in an unfavorable position, increasing the risk of sustaining an ACL (re)injury [9]. To date, research on muscle onset times has only been performed under non-fatigue conditions. Muscle onset patterns of ACLR and ACLD patients in response to fatigue are currently unknown, but could impact second ACL injury risk during activity.

Neuromuscular fatigue occurs during rigorous activity and may alter neuromuscular control and contribute to injury. Available literature demonstrates that fatigue alters quadriceps and hamstring muscle activation patterns in healthy individuals $[13,14]$. In patients after ACLR, Lepley et al. reported decreased activity of the lateral hamstring (LH) and vastus lateralis (VL) muscles post-fatigue [15]. However, Lessi and Viadanna observed increased activation of the VL, lateral hamstrings (LH) and gluteus maximus in patients after ACLR during the landing phase post-fatigue [16]. In ACLD patients, lower quadriceps muscle activity during walking, named "quadriceps avoidance gait" [17], higher vastus medialis muscle activity in running [18], decreased hamstrings activity during walking [19], and increased gastrocnemius muscle activity [8] have been observed. This muscle-activation strategy adopted by ACLD patients is hypothesized to occur to provide additional dynamic knee-joint stability [20].

Return to sport is a common goal of patients after ACL rupture. As fatigue is inevitable in sport, understanding its impact on neuromuscular compensation strategies in ACLR and ACLD patients compared to healthy controls is required so that rehabilitation can be more specific. Limited evidence is available regarding neuromuscular adaptation mechanisms induced by fatigue in ACLR patients. All studies in ACLD patients have been performed under non-fatigue conditions; therefore, there is no information about the effect of fatigue on muscle activity patterns in ACLD individuals. Therefore, the aim of this study was to evaluate the effects of fatigue on muscle activity patterns during single-leg drop landing in ACLR and ACLD patients compared to healthy controls. We hypothesized that ACLR and ACLD patients would have different muscle-activation patterns compared to healthy individuals in response to fatigue. In addition, we hypothesized that the response to fatigue would not be different between ACLR and ACLD patients.

\section{Materials and Methods}

\subsection{Subjects}

Twelve males 18-36 months post-ACLR, 12 males 18-36 months after ACL rupture (ACLD) and an additional 12 matched control subjects participated in this study (Table 1). A priori power analysis of fatigue studies indicated a minimum of 10 patients for each group to achieve a power of 0.8 [15,21]. Inclusion criteria required that all patients were: (1) males; (2) exercised for at least 3 sessions per week and 30 min per session; and (3) were between 18 and 30 years old. For ACLR and ACLD individuals, they had to participate in Level I or II sports after injury/reconstruction [22] (Table 2). Finally, ACLD patients had Grade 2 or 3 rupture and were required to meet the operational definition of a coper: athletes who were ACLD for at least 18 months with no symptoms of knee instability during regular participation in Level I and II sports. Individuals were excluded from participation if they: (1) were not medically cleared by their physician to participate in exercise; (2) had a history of bilateral ACL injury or injury to the medial collateral ligament, posterior cruciate ligament, lateral collateral ligament or meniscus in the contralateral and ipsilateral knee; (3) had any lower extremity injury episodes in the past 6 months that left them unable to participate in physical activity for more than 3 consecutive days; and (4) had a history of more than 1 ACL injury. Control subjects were excluded if they had: (1) a history of ACL injury or reconstruction; (2) current pain in either knee; or (3) sustained a lower extremity injury in the previous 6 months. This study was approved by the university institutional review board, and all subjects provided informed written consent before testing. 
Table 1. Subject demographics.

\begin{tabular}{|c|c|c|c|c|}
\hline & ACLD & ACLR & Control & \multirow{2}{*}{$p$} \\
\hline & Mean \pm SD & Mean \pm SD & Mean \pm SD & \\
\hline Age (year) & $24.5 \pm 2.32$ & $23.83 \pm 5.49$ & $24.92 \pm 2.81$ & 0.78 \\
\hline Height $(\mathrm{cm})$ & $174.5 \pm 4.62$ & $175.25 \pm 4.78$ & $175.00 \pm 5.23$ & 0.77 \\
\hline Mass (kg) & $75.25 \pm 7.13$ & $76.45 \pm 5.93$ & $74.75 \pm 7.50$ & 0.65 \\
\hline Months since surgery or initial injury & $23.25 \pm 6.95$ & $23.75 \pm 6.30$ & NA & \\
\hline Graft type & NA & $\begin{array}{c}\text { PT }=4 \\
\text { STG }=6 \\
\text { Allograft }=2\end{array}$ & NA & \\
\hline Injury grade & $\begin{array}{l}\text { Grade 2 }=7 \\
\text { Grade 3 }=5\end{array}$ & NA & NA & \\
\hline
\end{tabular}

Legend: $\mathrm{ACL}=$ anterior cruciate ligament, $\mathrm{ACLR}=$ anterior cruciate ligament reconstructed, $\mathrm{ACLD}=$ anterior cruciate ligament deficit, $\mathrm{SD}=$ standard deviation, NA = not applicable, $\mathrm{PT}=$ patellar tendon, $\mathrm{STG}=$ semitendinosus/gracilis.

Table 2. Activity level classification [22]. Modified to Iranian sport activities.

\begin{tabular}{ccc}
\hline Level & Sports Activity & Occupation Activity \\
\hline I & Jumping, cutting, pivoting (soccer, team & handball, basketball) \\
& Lateral movements, less pivoting than Level I (racket & Activity comparable to Level I sports \\
II & sports, martial arts, wrestling, gymnastics, aerobics) & Heavy manual labor, working on \\
& Straight ahead activities, no jumping or pivoting & uneven surfaces \\
III & (running, mountaineering, weightlifting) & Light manual work \\
IV & Sedentary & Activities of daily living \\
\hline
\end{tabular}

\subsection{Experimental Design}

This cross-sectional study had 2 independent variables: group (ACLR, ACLD and control) and fatigue state (pre-fatigue and post-fatigue). The main outcome measures were knee joint muscle activity onset times and reactive muscle activity of the vastus lateralis $(\mathrm{VL})$, vastus medialis $(\mathrm{VM})$, lateral hamstring $(\mathrm{LH})$, medial hamstring $(\mathrm{MH})$, lateral gastrocnemius (LG) and medial gastrocnemius (MG).

\subsection{Testing Procedures}

To record muscle activity, the skin for each electrode site was shaved and cleaned with isopropyl alcohol. Surface EMG (ME6000-T16, Megawin, Mega Electronics Ltd, Kuopio, Finland) electrodes with a preamplifier gain of 305 , a common mode rejection ration of $110 \mathrm{db}$, input impedance $>10 \mathrm{M} \Omega$ at $100 \mathrm{~Hz}$ and a 20-mm interelectrode distance were secured over the muscle bellies of the VL, VM, LH, MH, LG and MG muscles according to the technique described by SENIAM (Surface Electromyography for the Non-invasive Assessments of Muscles). Raw EMG data were sampled at $2000 \mathrm{~Hz}$ during dynamic activity and also during maximal voluntary isometric contractions (MVICs) for the purposes of normalization. Hamstring MVICs were performed while subjects were in prone position with knee in $30^{\circ}$ of flexion. Quadriceps MVICs were performed while subjects were seated with the knee in $90^{\circ}$ of flexion. Resistance was applied proximally to the ankle by the examiner while the subject performed $1 \mathrm{MVIC}$, either flexing (hamstrings) or extending (quadriceps) the knee as hard as possible. For gastrocnemius MVIC, resistive plantar flexion was performed in standing position (resisted heel raise movement). MVICs were repeated 3 times ( $5 \mathrm{~s}$ each) for each subject with 1 min rest between repetitions. Intraclass correlation coefficients (ICC) for the MVIC variables in the present study revealed good reliability ranging from $0.877-0.956$.

After the collection of the MVICs, subjects were instructed how to perform the dynamic landing task and were allowed adequate practice trials for familiarization. The landing task required subjects to perform a single leg vertical drop landing from a 30-cm box and land on a single leg on a force plate (Advanced Medical Technology, Inc., Watertown, MA, USA) sampling at $2000 \mathrm{~Hz}$ (Figure 1). The injured limb of ACLR and ACLD individuals and the dominant limb of controls were tested. Dominant limb was 
defined as the limb with which the participant would kick a ball. Three good trials, defined as the proper limb landing completely on the force platform, were analyzed (pre-fatigue trials).
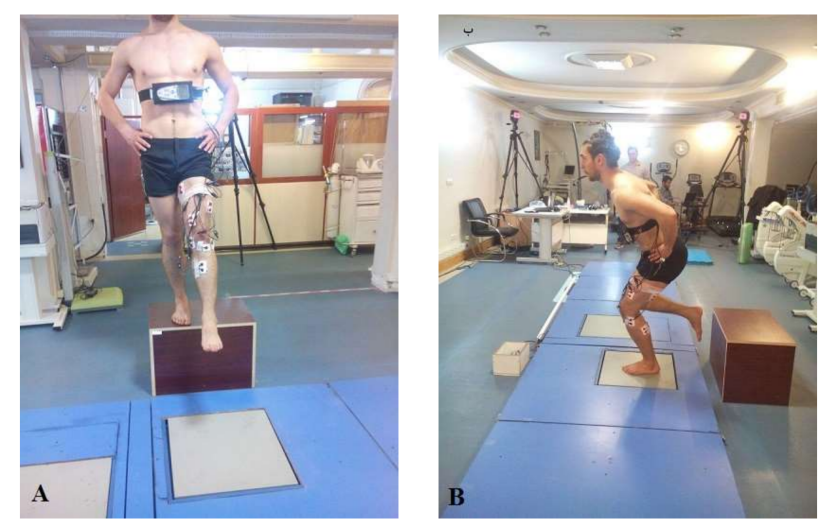

Figure 1. Single-leg vertical drop landing. (A) Preparation phase; (B) Landing phase.

Immediately after the pre-fatigue trials, subjects began the fatigue protocol. To induce fatigue, subjects were required to perform sets of 10 double-leg squats. They were asked to squat to approximately $90^{\circ}$ of knee flexion at a self-selected pace and without resistance, while the investigator provided continuous verbal feedback regarding knee-joint flexion angle during the fatiguing exercise [15,23-25]. After each set of 10 squats, subjects were required to perform 3 dynamic landings, similar to the pre-fatigue landing trials. Subjects continued performing the sets of squats followed by dynamic landings until maximal fatigue was achieved, defined as the point at which they could no longer perform 5 consecutive squats to $90^{\circ}$ of knee flexion. The subjects were asked to give a rating of perceived exertion (RPE) for the local fatigue in the lower extremities. The RPE is a subjective scale ranging from 6-20, where 6 means "no exertion at all" and 20 means "maximal exertion" [24]. If a subject reached maximum fatigue and could not safely complete the post-fatigue trials, then the 3 trials immediately before maximal fatigue were used for analysis.

\subsection{Data Analysis}

All EMG data were processed using MATLAB (MathWorks, Natick, MA, USA). Raw EMG data were band-pass filtered using a fourth-order, zero-lag Butterworth filter with high- and low-pass cutoff frequencies of 10 and $500 \mathrm{~Hz}$, respectively. EMG data were then full-wave rectified and processed using a root-mean-square algorithm with a 50-ms moving window. Dynamic EMG data recorded during the landing task were normalized to the peak muscle activity recorded during the MVIC. Muscle activity onset time was determined using the threshold method. A muscle was considered active when activity was at least two standard deviations above baseline for at least $30 \mathrm{~ms}$ [11]. Mean reactive muscle activity was analyzed from initial ground contact to $250 \mathrm{~ms}$ after ground contact [18].

\subsection{Statistical Analysis}

We used $3 \times 2$ repeated-measures analyses of variance (ANOVAs) to detect interactions, as well as the main effects of group (ACLR, ACLD and control) and fatigue state (pre-fatigue, post-fatigue) on muscle activity onset times and reactive muscle activity. Univariate tests including one-way ANOVA, and paired $t$-tests were used to determine group and fatigue state differences in the presence of significant interactions and main effects. 


\section{Results}

\subsection{Muscle Activity Onset Time}

There was a significant group $\times$ fatigue state interaction for VL $(F=4.70, p=0.016)$ and $\mathrm{LH}$ $(F=3.87, p=0.031)$ onset times. Further, there was a significant main effect of group for VL $(F=3.35$, $p=0.045), \mathrm{LH}(F=3.89, p=0.03)$ and $\mathrm{MH}(F=3.63, p=0.037)$ onset times. Post hoc analysis revealed that VL, LH and MH muscles turned on earlier in the ACLR compared to the control group post-fatigue $(p<0.05)$.

Muscle onset times did not differ between the ACLR and ACLD groups or the ACLD and control groups (Table 3). There was not a significant main effect of fatigue for any muscle tested.

Table 3. EMG onset times (ms pre-contact) at pre- and post-fatigue.

\begin{tabular}{ccc}
\hline \multirow{2}{*}{ Muscles } & Pre-Fatigue & Post-Fatigue \\
\cline { 2 - 3 } & Mean \pm SD & Mean \pm SD \\
\hline VM & & \\
ACLD & $168.21 \pm 85.23$ & $121.53 \pm 33.47$ \\
ACLR & $119.77 \pm 97.39$ & $167.58 \pm 81.98$ \\
Control & $101.22 \pm 57.17$ & $100.03 \pm 38.06$ \\
\hline VL $^{\text {a,b }}$ & & \\
ACLD & $122.03 \pm 55.81$ & $107.62 \pm 47.81$ \\
ACLR & $111.65 \pm 83.06$ & $194.79 \pm 119.35$ \\
Control & $89.16 \pm 55.41$ & $95.04 \pm 44.69$ \\
\hline MH ${ }^{b}$ & & \\
ACLD & $108.93 \pm 56.87$ & $134.41 \pm 38.65$ \\
ACLR & $148.65 \pm 81.18$ & $163.79 \pm 85.59$ \\
Control & $99.46 \pm 36.21$ & $100.77 \pm 35.19$ \\
\hline LH ${ }^{a}, b$ & & \\
ACLD & $186.00 \pm 99.75$ & $158.13 \pm 61.82$ \\
ACLR & $142.10 \pm 92.79$ & $227.75 \pm 104.99$ \\
Control & $117.42 \pm 42.36$ & $125.60 \pm 50.57$ \\
\hline MG & & \\
ACLD & $214.95 \pm 83.03$ & $242.09 \pm 93.05$ \\
ACLR & $286.83 \pm 99.33$ & $335.06 \pm 123.59$ \\
Control & $239.36 \pm 70.95$ & $270.65 \pm 128.80$ \\
\hline LG & & \\
ACLD & $167.81 \pm 71.61$ & $203.89 \pm 89.36$ \\
ACLR & $186.91 \pm 101.06$ & $193.90 \pm 79.94$ \\
Control & $208.02 \pm 69.33$ & $179.54 \pm 74.57$ \\
\hline
\end{tabular}

Legend: $\mathrm{ACLR}=$ anterior cruciate ligament reconstructed, $\mathrm{ACLD}=$ anterior cruciate ligament deficit, $\mathrm{SD}=$ standard deviation, $\mathrm{VM}=$ vastus medialis, $\mathrm{VL}=$ vastus lateralis, $\mathrm{MH}=$ medial hamstring, $\mathrm{LH}=$ lateral hamstring, $\mathrm{MG}=$ medial gastrocnemius, $\mathrm{LG}=$ lateral gastrocnemius. ${ }^{\text {a }}$ Indicates significant interaction of group $\times$ fatigue state.

${ }^{b}$ Indicates significant effect of group (ACLR vs. control).

\subsection{Reactive Muscle Activity}

There were significant fatigue state $(F=9.72, p=0.004)$ and group $(F=5.59, p=0.008)$ main effects for LG activity and a significant group $(F=3.39, p=0.041)$ main effect for VM activity. Specifically, fatigue decreased LG muscle activity in the ACLD and control groups. Further, ACLR patients had significantly less LG muscle activity than controls regardless of fatigue state. ACLD patients used more VM activity compared to the other groups post-fatigue. No group or fatigue effects were noted for VL, MH, LH and MG muscle activity (Figure 2). Furthermore, the interaction of group $\times$ fatigue state on muscle activity was not significant for any muscles. 


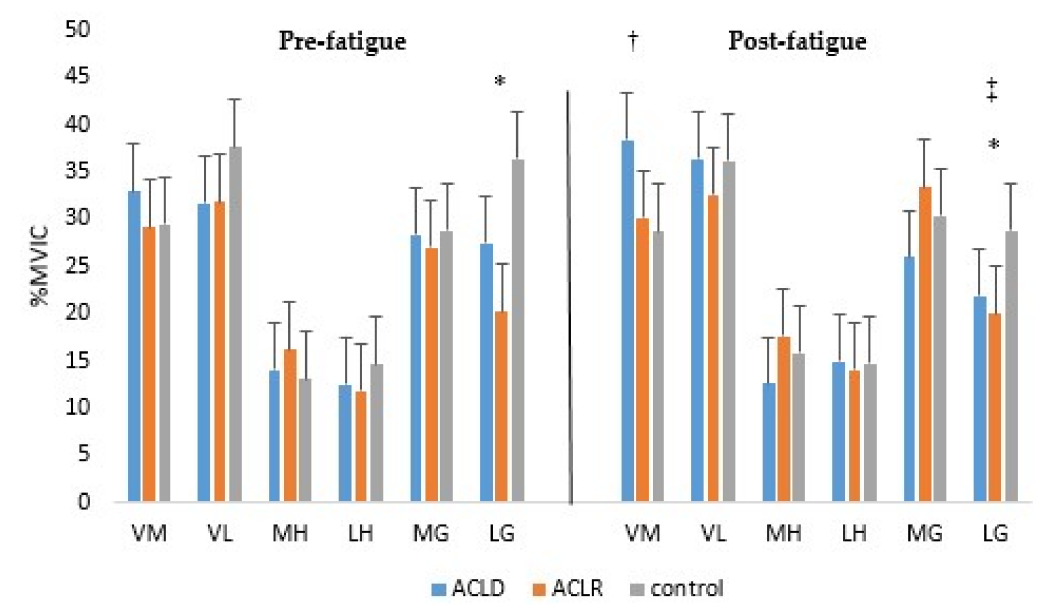

Figure 2. Reactive muscle activity at pre- and post-fatigue. * Indicates significant effect of group (ACLR vs. control regardless of fatigue state). ${ }^{\dagger}$ Indicates significant effect of group (ACLD vs. ACLR and control). ${ }^{\ddagger}$ Indicates significant effect of fatigue for ACLD and control.

\section{Discussion}

This research was conducted to determine the effects of fatigue on muscle activity patterns of ACL injured (ACLR and ACLD) patients compared to controls. This study is the first to demonstrate different muscle activity adaptations to fatigue in ACLR compared to ACLD patients.

Patients after ACLR demonstrated an active strategy to compensate for fatigue, in which they turned their muscles on earlier prior to landing [26]. This active strategy was specifically observed in the VL, $\mathrm{LH}$ and MH muscles of the ACLR group, which turned on significantly earlier compared to controls post-fatigue. Earlier activity of the LH has been reported previously during a drop jump [27]. Muscle pre-activity increases the sensitivity of the muscle spindles, allowing joint perturbation to be detected more quickly [28] and, thus, potentially responded to before injury can occur. Specifically, earlier onset times of the hamstrings post-fatigue could help decrease anterior tibial shear force on landing [18]. Early activation of the VL has been associated with higher levels of knee functionality for patients after ACLR; however, quadriceps activity may contribute to the characteristic straighter knee and decreased knee flexor moment at initial contact and during early stance observed in ACLR patients [29-31].

The ACLD group did not demonstrate different muscle onset times compared to the other groups or as a result of fatigue state. Previous investigations have shown earlier hamstrings, quadriceps and gastrocnemius activity onset times in ACLD patients compared to controls in a non-fatigued state $[8,10,32,33]$. Differences in task performed across studies may help explain the discrepancy. Previous work has examined muscle activity onset during walking [8] or single-leg hopping [10,32], while we utilized the single-leg drop jump, which does not require cyclical muscle activity as is observed in walking or muscle activity in order to complete take-off and landing phases as is required by the single-leg hopping task.

Patients in the ACLD group did demonstrate significantly more VM activity compared to ACLR and control groups post-fatigue (Figure 2). Although some investigators have found lower levels of quadriceps electromyographic activity during weight acceptance in ACLD individuals consistent with a "quadriceps avoidance gait" [17], most have not [11,18,34]. Thus, our results seem to confirm that patients do not avoid using their quadriceps during activity. Rather, increased VM activity may be an attempt to control knee joint deceleration during landing [18].

Fatigue resulted in a significant decrease of LG muscle activity in ACLD subjects and controls.

Less muscle activity post-fatigue may be the result of an inhibitory reflex that is evoked in the gastrocnemius muscle [35]. Alternatively, decreased LG activity after fatigue in the ACLD group may represent a lower reliance on the ankle-dominant strategy and greater reliance on the knee-dominant strategy (increased quadriceps activity) for stability [36]. However, there is limited evidence available 
to support these speculations and limited data on the effects of fatigue in ACLD participants. Therefore, it appears that more research is necessary to better understand the influence of fatigue on muscle activity of ACLD individuals.

ACLR patients used less LG muscle activity compared to controls regardless of fatigue state. In addition, the results indicate that ACLD individuals use lower levels of LG muscle activity compared to controls, but this finding was not statistically significant $(p=0.076)$. Previous researchers have demonstrated that the gastrocnemius probably plays an important role in the ACLD knee [11,33]. In fact, the gastrocnemius muscle is an antagonist of ACL, and its contraction can strain the ACL [37]; thus, lower LG muscle activity of LG may decrease strain on the ACL.

Fatigue had no influence on hamstrings muscle activity. We expected to observe greater muscle activity post-fatigue in ACLD and ACLR patients as a protective mechanism to reduce anterior tibial translation after injury [15,18]. Previous researchers have shown both decreased [14,19] and increased [16] hamstrings muscle activity post-fatigue in ACL injured patients. It is possible that our fatigue protocol emphasized fatigue of other muscle groups (quadriceps and gastrocnemius) over the hamstrings or that vertical drop landing is not a hamstrings-dependent task. Future investigations would benefit from examination of other fatiguing exercises or dynamic activities to enhance our understanding of how the hamstrings are influenced by fatigue in patients after ACLR and in those who are ACLD.

This study was not without limitations. Individuals who underwent patellar tendon $(n=4)$, semitendinosus/gracilis $(n=6)$ and allograft $(n=2)$ ACLR were included in this study and may have affected our results. While this increases the generalizability of our findings, future investigations are warranted to determine the potential differences in muscle activity between graft types. Additionally, the fatigue protocol used in this investigation did not simulate sport participation. Although our fatigue protocol incorporated a double-leg squatting motion and landings similar to movement that would be performed during activity, it is plausible that ACL injured subjects may respond differently to fatigue during an actual sporting event than when fatigue is induced in a controlled laboratory setting. Although all participants had similar demographics and physical activity participation, this does not verify equivalent aptitude for the vertical drop landing task among samples. Some patients may have demonstrated superior proficiency than others with the execution of vertical drop landing, and this could have influenced drop landing muscle activity. However, all participants were afforded practice trials, and feedback on correctly performing the task was provided to improve consistency of task performance across groups. Finally, only males were included in our study, and we cannot translate our results to females.

\section{Conclusions}

ACLR patients had earlier muscle activity onset times compared to controls post-fatigue. Thus, rehabilitation needs to focus on the pre-landing phase in order to optimize neuromechanical outcomes for patients after ACLR. ACLD patients rely more on reactive muscle activity (especially in quadriceps and gastrocnemius muscles) to establish knee joint stability post-impact. Increased VM and decreased LG activity post-fatigue warrants more focus on these muscles for ACLD patients in rehabilitation programs.

Acknowledgments: This study was implemented by the cooperation with the University of Tehran and Sports Sciences Research Institute of Iran.

Author Contributions: Mohammad Hossein Alizadeh and Hooman Minoonejad were the supervisor and advisor of this study, respectively, and helped in the writing and data gathering. Hamidreza Yazdi, as a knee surgeon, provided study subjects and identified inclusion and exclusion criteria for injured subjects. Abbey Thomas edited the paper technically and contributed to the final report. All authors contributed to the data analysis.

Conflicts of Interest: The authors declare no conflict of interest.

\section{Abbreviations}

ACLR Anterior cruciate ligament reconstructed

ACLD Anterior cruciate ligament deficit 


$\begin{array}{ll}\text { NA } & \text { Not applicable } \\ \text { PT } & \text { Patellar tendon } \\ \text { STG } & \text { Semitendinosus/gracilis } \\ \text { VM } & \text { Vastus medialis: } \\ \text { VL } & \text { Vastus lateralis } \\ \text { MH } & \text { Medial hamstring } \\ \text { LH } & \text { Lateral hamstring } \\ \text { MG } & \text { Medial gastrocnemius } \\ \text { LG } & \text { Lateral gastrocnemius. }\end{array}$

\section{References}

1. Ardern, C.L.; Webster, K.E.; Taylor, N.F.; Feller, J.A. Return to sport following anterior cruciate ligament reconstruction surgery: A systematic review and meta-analysis of the state of play. Br. J. Sports Med. 2011, 45, 596-606. [CrossRef] [PubMed]

2. Gobbi, A.; Francisco, R. Factors affecting return to sports after anterior cruciate ligament reconstruction with patellar tendon and hamstring graft: A prospective clinical investigation. Knee Surg. Sports Traumatol. Arthrosc. 2006, 14, 1021-1028. [CrossRef] [PubMed]

3. Myklebust, G.; Holm, I.; Mæhlum, S.; Engebretsen, L.; Bahr, R. Clinical, functional, and radiologic outcome in team handball players 6 to 11 years after anterior cruciate ligament injury: A follow-up study. Am. J. Sports Med. 2003, 31, 981-989. [CrossRef] [PubMed]

4. Strehl, A.; Eggli, S. The value of conservative treatment in ruptures of the anterior cruciate ligament (ACL). J. Trauma Acute Care Surg. 2007, 62, 1159-1162. [CrossRef] [PubMed]

5. Blagojevic, M.; Jinks, C.; Jeffery, A.; Jordan, K. Risk factors for onset of osteoarthritis of the knee in older adults: A systematic review and meta-analysis. Osteoarthr. Cartil. 2010, 18, 24-33. [CrossRef] [PubMed]

6. Luc, B.; Gribble, P.A.; Pietrosimone, B.G. Osteoarthritis prevalence following anterior cruciate ligament reconstruction: A systematic review and numbers-needed-to-treat analysis. J. Athl. Train. 2014, 49, 806-819. [CrossRef] [PubMed]

7. Di Stasi, S.; Myer, G.D.; Hewett, T.E. Neuromuscular training to target deficits associated with second anterior cruciate ligament injury. J. Orthop. Sports Phys. Ther. 2013, 43, 777-792. [CrossRef] [PubMed]

8. Lass, P.; Kaalund, S.; Iefevre, S.; Arendt-Nielsen, L.; Sinkjæ, T.; Simonsen, O. Muscle coordination following rupture of the anterior cruciate ligament: Electromyographic studies of 14 patients. Acta Orthop. Scand. 1991, 62, 9-14. [CrossRef] [PubMed]

9. Gokeler, A.; Hof, A.; Arnold, M.; Dijkstra, P.; Postema, K.; Otten, E. Abnormal landing strategies after ACL reconstruction. Scand. J. Med. Sci. Sports 2010, 20, e12-e19. [CrossRef] [PubMed]

10. Klyne, D.M.; Keays, S.L.; Bullock-Saxton, J.E.; Newcombe, P.A. The effect of anterior cruciate ligament rupture on the timing and amplitude of gastrocnemius muscle activation: A study of alterations in EMG measures and their relationship to knee joint stability. J. Electromyogr. Kinesiol. 2012, 22, 446-455. [CrossRef] [PubMed]

11. Lindström, M.; Felländer-Tsai, L.; Wredmark, T.; Henriksson, M. Adaptations of gait and muscle activation in chronic ACL deficiency. Knee Surg. Sports Traumatol. Arthrosc. 2010, 18, 106-114. [CrossRef] [PubMed]

12. Solomonow, M.; Krogsgaard, M. Sensorimotor control of knee stability: A review. Scand. J. Med. Sci. Sports 2001, 11, 64-80. [CrossRef] [PubMed]

13. Rozzi, S.L.; Lephart, S.M.; Fu, F.H. Effects of muscular fatigue on knee joint laxity and neuromuscular characteristics of male and female athletes. J. Athl. Train. 1999, 34, 106. [PubMed]

14. Wojtys, E.M.; Wylie, B.B.; Huston, L.J. The effects of muscle fatigue on neuromuscular function and anterior tibial translation in healthy knees. Am. J. Sports Med. 1996, 24, 615-621. [CrossRef] [PubMed]

15. Lepley, L.K.; Thomas, A.C.; McLean, S.G.; Palmieri-Smith, R.M. Fatigue's Lack of Effect on Thigh-Muscle Activity in Anterior Cruciate Ligament-Reconstructed Patients During a Dynamic-Landing Task. J. Sport Rehabil. 2013, 22, 83-92. [CrossRef] [PubMed]

16. Lessi, G.C.; Serrão, F.V. Effects of fatigue on lower limb, pelvis and trunk kinematics and lower limb muscle activity during single-leg landing after anterior cruciate ligament reconstruction. Knee Surg. Sports Traumatol. Arthrosc. 2017, 25, 2550-2558. [CrossRef] [PubMed] 
17. Berchuck, M.; Andriacchi, T.; Bach, B.; Reider, B. Gait adaptations by patients who have a deficient anterior cruciate ligament. J. Bone Jt. Surg. 1990, 72, 871-877. [CrossRef]

18. Swanik, C.B.; Lephart, S.M.; Giraldo, J.L.; DeMont, R.G.; Fu, F.H. Reactive muscle firing of anterior cruciate ligament-injured females during functional activities. J. Athl. Train. 1999, 34, 121. [PubMed]

19. Van Lent, M.; Drost, M.; van den Wildenberg, F. EMG profiles of ACL-deficient patients during walking: The influence of mild fatigue. Int. J. Sports Med. 1994, 15, 508-514. [CrossRef] [PubMed]

20. Chmielewski, T.L.; Hurd, W.J.; Rudolph, K.S.; Axe, M.J.; Snyder-Mackler, L. Perturbation training improves knee kinematics and reduces muscle co-contraction after complete unilateral anterior cruciate ligament rupture. Phys. Ther. 2005, 85, 740-749. [PubMed]

21. Webster, K.E.; Santamaria, L.J.; Mcclelland, J.A.; Feller, J.A. Effect of fatigue on landing biomechanics after anterior cruciate ligament reconstruction surgery. Med. Sci. Sports Exerc. 2012, 44, 910-916. [CrossRef] [PubMed]

22. Hefti, E.; Müller, W.; Jakob, R.; Stäubli, H.-U. Evaluation of knee ligament injuries with the IKDC form. Knee Surg. Sports Traumatol. Arthrosc. 1993, 1, 226-234. [CrossRef] [PubMed]

23. Borotikar, B.S.; Newcomer, R.; Koppes, R.; McLean, S.G. Combined effects of fatigue and decision making on female lower limb landing postures: Central and peripheral contributions to ACL injury risk. Clin. Biomech. 2008, 23, 81-92. [CrossRef] [PubMed]

24. Gokeler, A.; Eppinga, P.; Dijkstra, P.; Welling, W.; Padua, D.; Otten, E.; Benjaminse, A. Effect of fatigue on landing performance assessed with the landing error scoring system (less) in patients after ACL reconstruction: A pilot study. Int. J. Sports Phys. Ther. 2014, 9, 302. [PubMed]

25. Mclean, S.G.; Samorezov, J.E. Fatigue-induced ACL injury risk stems from a degradation in central control. Med. Sci. Sports Exerc. 2009, 41, 1661-1672. [CrossRef] [PubMed]

26. Horita, T.; Komi, P.; Nicol, C.; Kyröläinen, H. Interaction between pre-landing activities and stiffness regulation of the knee joint musculoskeletal system in the drop jump: Implications to performance. Eur. J. Appl. Physiol. 2002, 88, 76-84. [PubMed]

27. Pfeifer, K.; Banzer, W. Motor performance in different dynamic tests in knee rehabilitation. Scand. J. Med. Sci. Sports 1999, 9, 19-27. [CrossRef] [PubMed]

28. Dyhre-Poulsen, P.; Simonsen, E.B.; Voigt, M. Dynamic control of muscle stiffness and H reflex modulation during hopping and jumping in man. J. Physiol. 1991, 437, 287-304. [CrossRef] [PubMed]

29. Bryant, A.L.; Newton, R.U.; Steele, J. Successful feed-forward strategies following ACL injury and reconstruction. J. Electromyogr. Kinesiol. 2009, 19, 988-997. [CrossRef] [PubMed]

30. Di Fabio, R.; Graf, B.; Badke, M.; Breunig, A.; Jensen, K. Effect of knee joint laxity on long-loop postural reflexes: Evidence for a human capsular-hamstring reflex. Exp. Brain Res. 1992, 90, 189-200. [CrossRef] [PubMed]

31. Webster, K.E.; Wittwer, J.E.; O'brien, J.; Feller, J.A. Gait patterns after anterior cruciate ligament reconstruction are related to graft type. Am. J. Sports Med. 2005, 33, 247-254. [CrossRef] [PubMed]

32. Rudolph, K.; Axe, M.; Snyder-Mackler, L. Dynamic stability after ACL injury: Who can hop? Knee Surg. Sports Traumatol. Arthrosc. 2000, 8, 262-269. [CrossRef] [PubMed]

33. Sinkjær, T.; Arendt-Nielsen, L. Knee stability and muscle coordination in patients with anterior cruciate ligament injuries: An electromyographic approach. J. Electromyogr. Kinesiol. 1991, 1, 209-217. [CrossRef]

34. Ciccotti, M.G.; Kerlan, R.K.; Perry, J.; Pink, M. An electromyographic analysis of the knee during functional activities: II. The anterior cruciate ligament-deficient and-reconstructed profiles. Am. J. Sports Med. 1994, 22, 651-658. [CrossRef] [PubMed]

35. Woods, J.; Furbush, F.; Bigland-Ritchie, B. Evidence for a fatigue-induced reflex inhibition of motoneuron firing rates. J. Neurophysiol. 1987, 58, 125-137. [CrossRef] [PubMed]

36. Padua, D.A.; Arnold, B.L.; Perrin, D.H.; Gansneder, B.M.; Carcia, C.R.; Granata, K.P. Fatigue, vertical leg stiffness, and stiffness control strategies in males and females. J. Athl. Train. 2006, 41, 294. [PubMed]

37. Fleming, B.C.; Renstrom, P.A.; Ohlen, G.; Johnson, R.J.; Peura, G.D.; Beynnon, B.D.; Badger, G.J. The gastrocnemius muscle is an antagonist of the anterior cruciate ligament. J. Orthop. Res. 2001, 19, 1178-1184. [CrossRef]

(C) 2018 by the authors. Licensee MDPI, Basel, Switzerland. This article is an open access article distributed under the terms and conditions of the Creative Commons Attribution (CC BY) license (http:// creativecommons.org/licenses/by/4.0/). 